Journal of Environmental and Agricultural Studies

ISSN: $2710-140$

DOI: $10.32996 /$ jeas

Journal Homepage: www.al-kindipublisher.com/index.php/jeas

JAES

\title{
Seasonal Population Abundance of Bactrocera dorsalis Hendel (Diptera: Tephritidae) in Selected Districts of Northern KwaZulu Natal, South Africa
}

\author{
Simphiwe Mnguni 8 (D). \\ Department of Agriculture, Land Reform and Rural Development (DALRRD), Directorate: Plant Health, Division: Early Warning \\ Systems, Private Bag x 14. Pretoria, 0001. South Africa \\ $\triangle$ Corresponding Author: Simphiwe Mnguni, E-mail: simphiwemnguni.sm@gmail.com
}

\section{ARTICLE INFORMATION}

Received: April 08, 2021

Accepted: June 02, 2021

Volume: 2

Issue: 1

DOI: 10.32996/jeas.2021.2.1.8

\section{KEYWORDS}

Bactrocera dorsalis, Tephritidae; Bactrocera; South Africa; KwaZulu Natal,quarantine

\section{ABSTRACT}

The fruitfly Bactrocera dorsalis is an economically important pest that requires management for the sustainability of agriculture in South Africa. This pest has spread from the country's Northern parts within a decade, where it has completely established and spread to other neighbouring provinces. The pest spreads at various rates due to different biotic and abiotic factors. Understanding factors that influence pest prevalence will assist with practical management strategies. Temperature is one of the factors that assist the invasive potential of $B$. dorsalis. KwaZulu Natal province is among the hotspot provinces in the country besides Limpopo and Mpumalanga. Trap catches in Northern KwaZulu Natal, uMkhanyakude and King Cetshwayo districts show that the seasonal populations of $B$. dorsalis are continuously present all year round, even with the application of management options to reduce pest populations. Host availability plays a significant role in the invasion and total outcompeting of other native fruitflies e.g. Ceratitis punctata.

\section{Introduction}

The introduction of fruitfly Bactrocera dorsalis in South Africa was first detected in 2010. Bactrocera dorsalis is an invasive pest of quarantine and economic importance that was reported in Africa around 2003 and originated from Sri Lanka (Mwatawala et al., 2004; Drew et al., 2005). The spread of $B$. dorsalis has been rapid in Africa, affecting over 28 other African countries (Drew et al., 2005; Ekesi \& Billah, 2006). In South Africa, the occurrence of $B$. dorsalis has always been of concern from early warning systems (EWS) based on risk analysis, monitoring, awareness and communication and response capability. Based on EWS $B$. dorsalis had the potential to invade South Africa which was a threat that required biosecurity measures to ensure that damage to crops is minimized (Kriticos et al., 2013). Bactrocera dorsalis is a highly invasive species and very destructive to crop yield for farmers of various fruits, vegetables, and nuts worldwide.

\section{Literature review}

The larval stage in the life cycle of $B$. dorsalis is the most damaging to fruits because of larval feeding on the soft flesh of fruits. After ovipositing occurs by a female fly, the larvae develop under the skin of the fruit or soft tissues of the plant and begin to feed on the fruit or plant's flesh. Once feeding occurs, other microorganisms can invade the site of larval feeding and cause the fruit to decay faster. Although ripe fruits are believed to be preferred for ovipositing, unripe fruits have served as hosts for eggs as well (Ekesi \& Billah, 2007; James \& Schiffers, 2007). Development from egg to adult under summer conditions requires about 16 days. The mature larva emerges from the fruit, drops to the ground, and forms a tan to dark brown puparium. Pupation occurs in the soil. About nine days are required for the attainment of sexual maturity after the adult fly emerges. The developmental periods may be extended considerably by cool weather. Under optimum conditions, a female can lay more than 3,000 eggs during her lifetime, but under field conditions, from 1,200 to 1,500 eggs per female is the usual production. Apparently, ripe fruit is preferred for oviposition, but immature ones may also be attacked.

\section{K C AL-KINDI CENTER \\ $R$ DFOR RESEARCH AND}

Your gateway to world-class research

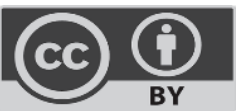

Published by Al-Kindi Center for Research and Development, London, United Kingdom. Copyright (c) the author(s). This open access article is distributed under a Creative Commons Attribution (CC-BY) 4.0 license 
The concern for $B$. dorsalis is mainly its polyphagous nature, affecting over 13 plant families. The losses associated with $B$. dorsalis in Africa have led to annual economic damage of US\$42 million and US\$1 billion worldwide (Motswagole et al., 2019). The presence of $B$. dorsalis posed a significant risk to South Africa due to the presence of many hosts that could suffer damage, which some are part of the export markets. Within a decade, B. dorsalis has spread from the country's Northern parts, where it has completely established and spread to other neighbouring provinces. This pest is classified as a quarantine pest for South Africa; and by many other countries in the world given the damage it causes and the significance for the export of host material (DeVilliers et al., 2016). The presence of $B$. dorsalis requires strict phytosanitary regulations to minimize its spread and establishment. Understanding factors that influence pest abundance is important for effective pest management control. The $B$. dorsalis population dynamics are influenced by abiotic and biotic factors (Ganie et al., 2013). Of the crucial factors for growth and pest population abundance is the temperature (Choi, 2020).

Previous climatic models indicated how temperatures in various parts of South Africa could be vulnerable to $B$. dorsalis (De Villiers et al., 2016). The impact of consistent high temperature ranging from 15 to $36^{\circ} \mathrm{C}$ contributes to the development, lifespan, and fecundity of $B$. dorsalis (Yang, 1994; Chen \& Ye, 2007, Choi, 2020). However, accidental introductions due to various scenarios have resulted in this pest being detected in other cold areas (in the cold season) of South Africa. An example: a notification was issued under the International Plant Protection Organisation (IPPC) in 2019 on B. dorsalis found close to a Malus spp. In Bethlehem, the Eastern Part of the Free State Province of South Africa is a region known to be cold in the country. After the delimiting surveys and phytosanitary actions in accordance with the South African Bactrocera dorsalis Fruit Fly (SABiFF) Action Plan to restrict the movement of the host material, no further detections were found (IPPC, 2019). Therefore, thermal factors may not be the only consideration for the spread of $B$. dorsalis. In contrast, other scholars have cited the possibility that $B$. dorsalis in Africa prefers relative humidity and high levels of rainfall (De Meyer et al., 2010; Vayssières et al., 2009; Wih, 2008). Finding B.dorsalis in areas where the minimum temperatures go below 9 degrees are close to the findings of Rwomushana et al. (2008b), who reported lower developmental thresholds of $8.8,9.4$ and $8.7^{\circ} \mathrm{C}$ for eggs, larvae and pupae, separately, for $B$. dorsalis in Africa. Finding B. dorsalis species in South Africa's area of very cold temperatures could indicate mutation with a new ability to withstand even colder temperatures.

While the fruitfly trapping system indicates species present and the population numbers, trapping systems play an important role in decisions concerning the need to implement suppression measures and monitor the population dynamics of fruitflies. The objective of this paper is to:

1. Indicate the seasonal abundance of fruitflies present in King Cetshwayo and uMkhanyakude districts in northern KwaZulu Natal.

2. Indicate the efficacy of the implemented suppression methods used.

\section{Method}

The trapping systems for fruitflies in South Africa relies on the International Standards of Phytosanitary Measures (ISPMs) such as ISPM No. 26 (Establishment of Pest-Free Areas for Fruit Flies (Tephritidae)), ISPM No. 30 (Establishment of Areas of Low Pest Prevalence for Fruit Flies (Tephritidae)) and ISPM No. 35 (Systems Approach for Pest Risk Management of Fruit Flies). The mentioned ISPM's describe the procedures, equipment e.g., traps and attractants, data recording and analysis.

Fruifly pest populations were monitored using lure-baited traps with attractants/lures, loaded with a killing strip (Vapona) into yellow McPhail bucket traps, which is an attractive colour for fruitflies. Mainly Biolure monitors male and female Bactrocera spp, catching Ceratitis spp. Methyl Eugenol (ME) is male-specific, capturing many species of the genus Bactrocera used as attractants/lures. Traps should be hung close to $1.8 \mathrm{~m}$ on trees, ensuring that the trap is not placed directly facing the sun but also free from foliage to ensure that fruitflies have sufficient access to the trap. Traps were hung for 4 weeks and specimens collected for counting and analysis. The trap location map below shows the actual trap placements in Northern KwaZulu Natal below (Fig.1). Traps were placed in shaded spots to avoid direct contact with the sun, where possible and clear spots were chosen to ensure easy access to the trap. Traps were placed in eThekwini and llembe districts along the transect of the highway, down to King Cetshwayo and Umkhanyakude districts from the 16 ${ }^{\text {th }}$ November 2010 to November 2020. Other districts in Northern KwaZulu Natal include uMzinyathi, Amajuba, Zululand (which are all lowland). These will be excluded in the study as the traps were only placed in 2020 and there is no significant pest counts for Bactrocera dorsalis detected in those regions (an average of fewer than 5 pests have been caught on traps). Generally, B. invadens are believed to be lowland pests (Vargas et al. 1983, Wong et al. 1985, Harris et al. 1986; Ekesi et al. 2006). 


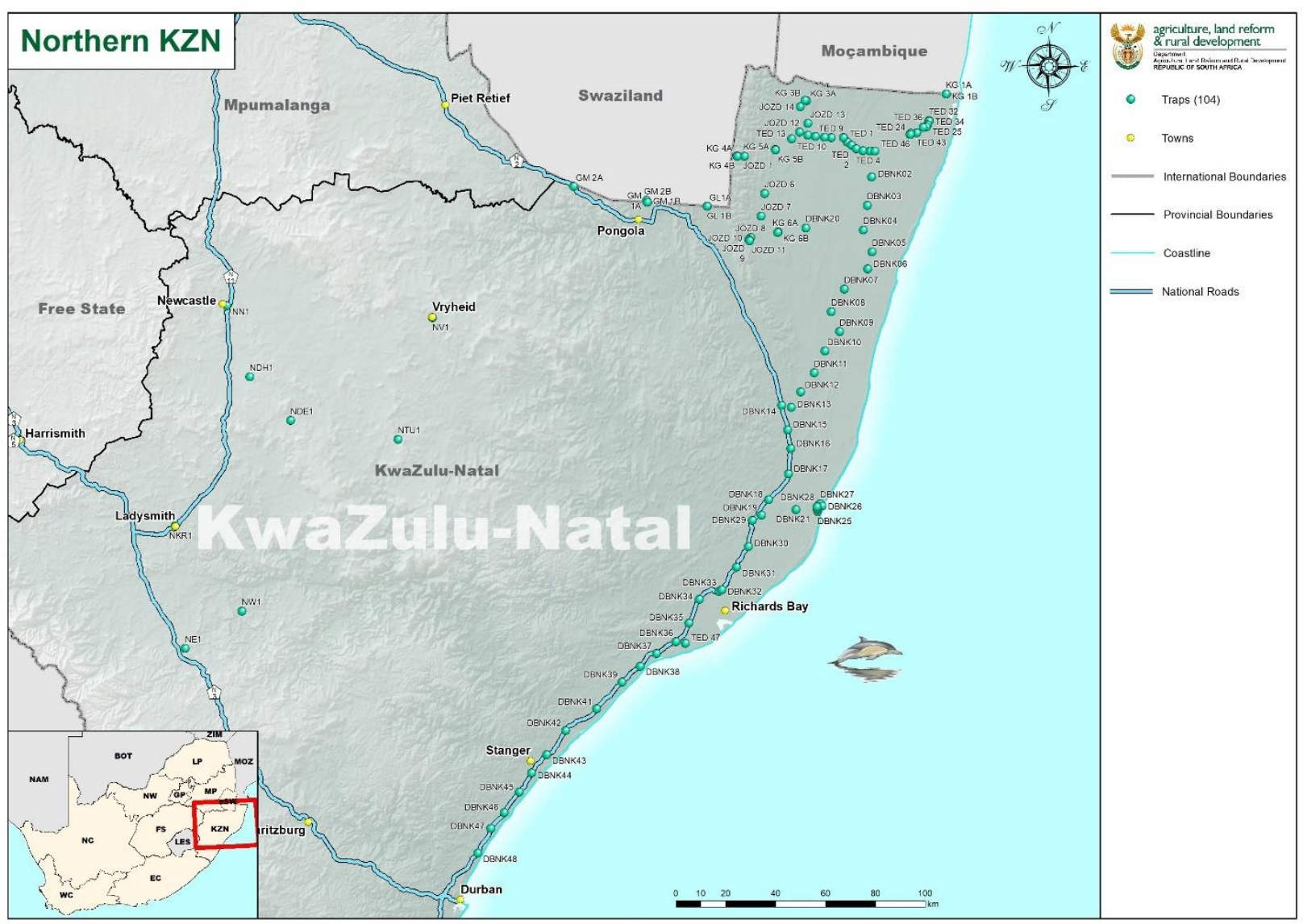

Figure 1: Map showing fruifly traps along with northern KwaZulu Natal.

Northern KwaZulu Natal experiences tropical temperatures (Allemann \& Young, 2006). The average maximum and minimum temperature range between 16 to $25^{\circ} \mathrm{C}$ in winter. During the summer months, temperatures range from 23 to $35^{\circ} \mathrm{C}$ (between September and April) and maximum relative humidity was 33 and 76\%, respectively. There are several tropical and subtropical crops such as marula (Sclerocarya birrea), monkey orange (Strychnos spinosa), bananas (Musa acuminata), avocado (Persia americana), papaya (Carica papaya) and mango (Mangifera indica) are found in abundance in the backyards, the streets and orchards. Deciduous crops found include peaches and nectarines (Prunus persica). Suitable areas for citrus e.g., oranges, grapefruit, lemons, soft citrus/mandarins include Nkwaleni, Pongola and Mkhuze (Allemann \& Young, 2006).

The quantity of caught fruitflies introduced in the trap will be presented as the flies per trap per day (FTD) of adults for a specified period. The traps are placed in the field and graphs. The FTD provides a comparative measure of the adult pest population in each existence, whereas graphs illustrate relationships in the data.

\section{Results and Discussion}

Servicing of traps could not occur every month due to several challenges and dynamics encountered throughout the years. The list of the challenges that may have contributed to non-service of traps in some certain months throughout the years included: staff shortages, field injuries, vehicle breakdowns due to rough terrain, procurement delays of stock from the government supply chain and recent lockdown due to COVID 19 pandemic which limited travelling.

With constant traps serviced, there were no B.dorsalis caught in the Northern KwaZulu Natal from 2010 from the first outbreak in South Africa until September 2013 with 14 pest counts found with a further increase of up to 19 species that were caught in November 2013. The $B$. dorsalis increase officially began in 2014 with the highest number of catches 1472 in April see Fig. 1 . In 2014 further species of Tephritidae were caught from the traps, including Ceratitis punctata with a count of 252 species found, with a majority of 232 caught in April and a minority of 20 caught in February. 


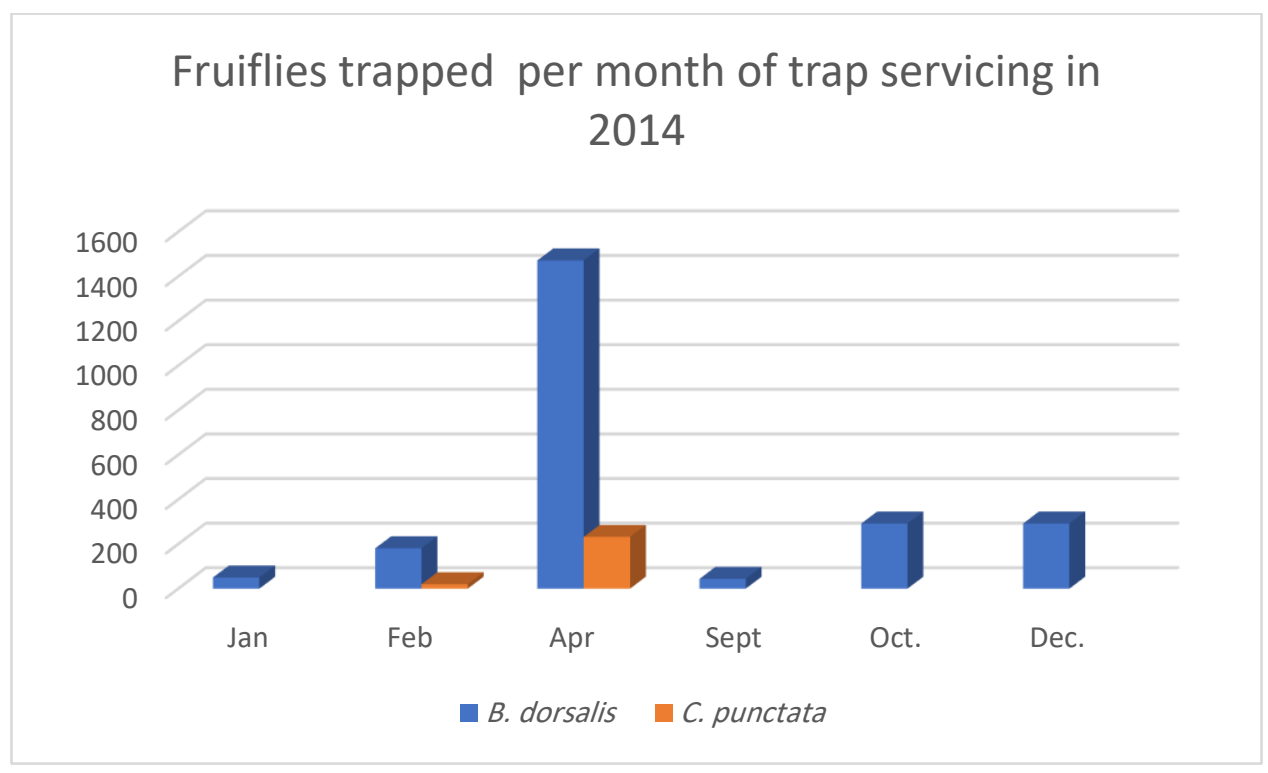

Figure 1: Showing fruitflies (B. dorsalis and C. punctata) caught in different months in 2014.

The data collected in 2015 indicated an exponential increase of species caught in the seven months of trap servicing see Fig. 2. In both 2014 and 2015 fruitflies pest counts were high in April when compared to the other months. With available results only for June 2016, species caught were 7120 , which was still relatively high considering the cold temperature.

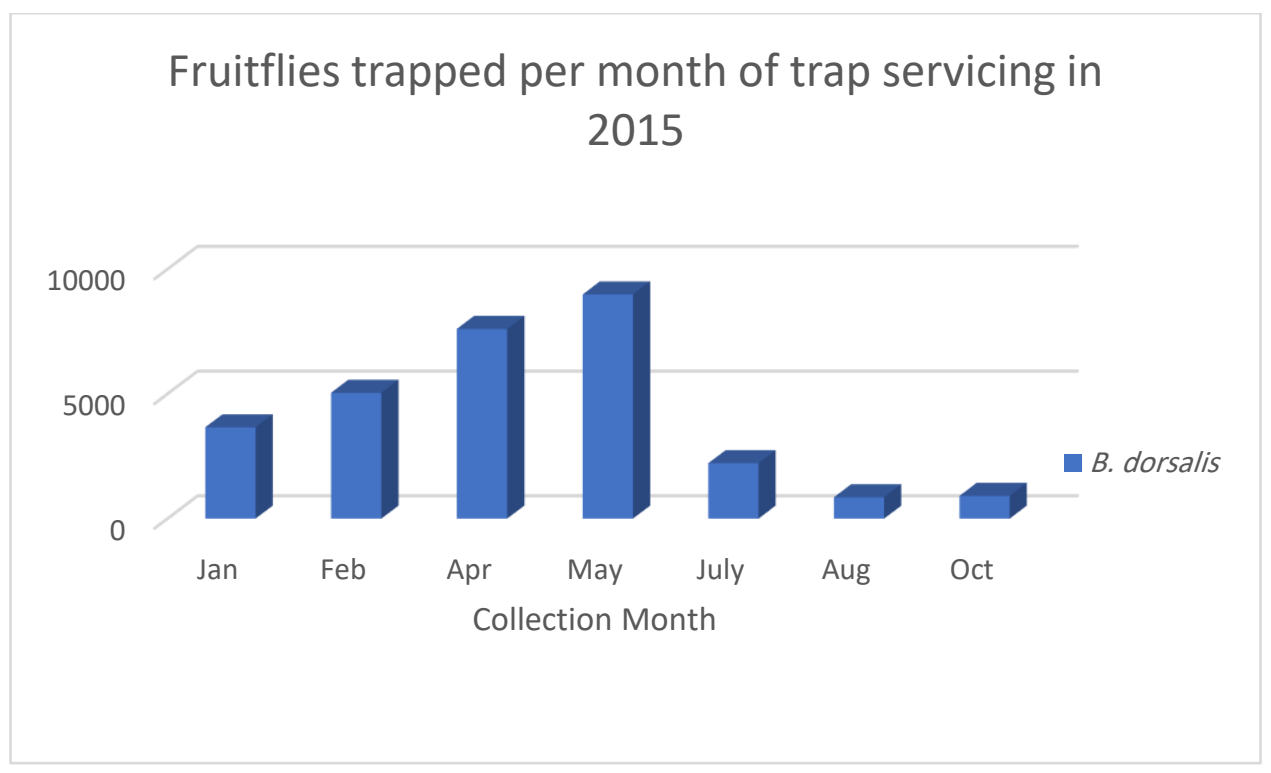

Figure 2: Showing fruitflies (B. dorsalis) caught in different months in 2015.

In the initial phase of fruitfly trapping in Northern KwaZulu Natal (King Cetshwayo and Mkhanyakude district), the indigenous fruitfly species were detected in traps along with $B$. dorsalis. The displacement of $C$. punctata took place in 2014 as no further pest counts were ever found years later. These findings are like the study of Ekesi et al. (2009), which found that the arrival of $B$. dorsalis displaced the indigenous fruit fly species Ceratitis cosyra (Walker), constituting 98 and $88 \%$ of the total population in traps, respectively. The displacement ability of $B$. dorsalis in KwaZulu Natal may be due to conducive high temperatures that allow the population to increase, together with the availability of host material. This information supports the importance of removing fallen fruit for the management of fruit fly populations in orchards and backyards.

Most importantly, the rapid displacement of $B$. dorsalis may be an indication that it is competitive for habitat. The aggressive existence of $B$. dorsalis been shown by Bruno et al. (2005). Many other studies such as those of Duyck et al. (2006) and Keiser et al. 
(1974) have also indicated that Ceratitis species tend to be outcompeted by Bactrocera species due to their abilities to protect oviposition sites on hosts and, most importantly, their embedded mobile ability.

Surveillance was not carried out in 2017 due to stock unavailability. In 2018 traps were also serviced in five varied months (July, August, September, October, November) as seen in Fig. 3. Trap catches for 2018 were almost constant between $966-1416$ pest catches. For 2019 traps were serviced for 6 months. Traps in 2019 were serviced in January, June, August, October, November, and December. The highest pest catches were in January and December, between $5860-6905$. The other months of 2019 had slightly higher pest catches between 1040-2281. In 2020 servicing was conducted in 10 months (all months except March and April) see Fig.3. In 2020, the highest number B. dorsalis catches was seen. In January, there were 23262 pest catches, which increased to 43 512 in February. In May 2019, was a subsiding number of pest catches to 16 467. The following months were almost similar in pest catches to 2018/2019 growing seasons from 1326-2566.

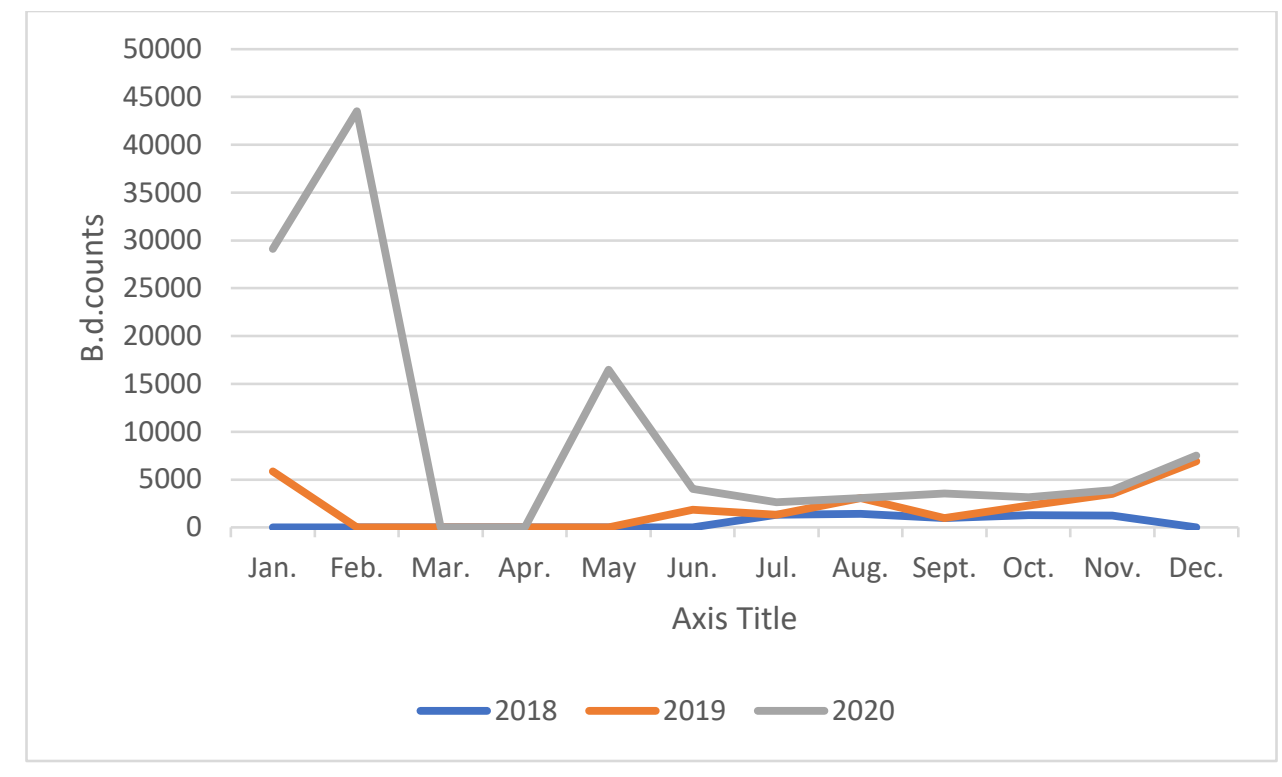

Figure 3: Showing fruitflies (B. dorsalis) catches between different months of 2018-2019.

Since 2014, April and May have the highest pest counts of all other months, largely due to ripened hosts, e.g. mangos highly abundant in the area and serve as a special host for B. dorsalis. Pest populations have increased from 2014 and flies are caught each month. This may be due to the presence of host material which ripens in different months. Between 2018 to 2020 December, January were also notably higher on pest counts due to conducive temperatures and ripe fruits. Most pest catches were found along with production areas, rural villages with host material instead of public roads, followed by fruit stalls. Within the selected districts of northern KwaZulu Natal B. dorsalis is well established and favored due to climatic conditions in those areas. The establishment of $B$. dorsalis in the selected districts of KwaZulu Natal suggests that the CLIMEX model of Stephens et al. (2007) and EPPO (2010) for climatic suitability in this area were correct. In some years, a lack of data collection could be overcome by outsourcing a service provider to conduct fruit fly surveillance. Outsourcing a service provider will solve procurement delays in government, assist with limited staff and ensure that data is readily available.

While chemicals such as GF120 and the use of the male annihilation technique (MAT) bait stations were distributed for use to farmers, in previous years, including 2018/2019 growing season, the fruitfly numbers continued to soar. Higher than above threshold fruitfly population indicate a great need for seemingly inadequate interventions in controlling the fruitflies in the selected areas. MAT is a strategy to lure and kill male flies with the aim is to reduce male fly populations which contribute to reduced mating opportunities with females upon feeding. Bait stations are usually placed in areas (perimeter of the property or orchard) of up to 20/hectare from Spring through to Autumn (e.g., August, November \& February). Bait stations need replacing every 3-4 months. Bait stations may need to be used even in the winter season in the case of this study area. Even though chemical use and calibration training was done, there is the ongoing need for support to ensure that it is accurately timed and MAT blocks are changed after a valid period.

Average captures in fly per trap per day (FTD) in uMkhanyakude and King Cetshwayo districts is above the FTD index of 0.5. The greatest population peak was indicated between 2019/2020 growing season. For the uMkhanyakude district in May, 2019 (FTD =6.9) with 9170 specimens in May, 2020 (FTD = 8.5), 12485 specimens were collected. For King Cetshwayo district May 2019 $($ FTD =1.3) with 1453 specimens, in May 2020 (FTD = 2.7), 3982 specimens were collected. This indicates a steady increase of fruifly population and a clear indication that uMkhanyakude district is the highly infested of the two districts. Most traps in King

Page | 83 
Cetshwayo district lie along the transect (N2 highway/public road), next to fruit stalls. In the uMkhanyakude district, most traps were placed along with production areas, rural villages with host material and those with the highest number of pest catches instead of public roads, followed by fruit stalls. Overall, fruitfly management control requires the integrated pest management (IPM) approach for varied tactics to optimize the control. Prospects of future introduction of biological controls towards $B$. dorsalis may need to be explored for use because chemicals yield limited results in this area.

\section{Conclusion}

To suppress B. dorsalis populations for sustainable management e.g. parasitoids (biological control) may need to be initiated. Fields trials for suitable biological controls may be required under the natural environmental conditions of the study area. Sponsorship may be necessary to ensure that pest-free areas are maintained in relation to $B$. dorsalis by using biological control agent at a large scale. Many farmers may also be lacking in management options as they only supply local markets. The offset of all the interventions is also the lack of proper sanitation along the fields which serves as a breeding ground for fruitfly population. Continued awareness efforts may be necessary to highlight the important need for minimizing fruitfly pest populations. The management strategies for $B$. dorsalis in the study area need to be improved. The results obtained were impossible to tell if the distributed chemicals were effective and used accurately to minimize fruitfly populations. Considering future climate change, it may be risky to keep the fruitfly populations high. Other opportunities offering different applications such as environment-friendly and residue-free strategies may need to be adopted to ensure food security. This study shows a wide variation of area-wide IPM application as opposed to individual orchard IPM approach on effort and yielding results.

\section{Acknowledgements}

A dedicated team largely collected data from Inspection Services under the Department of Agriculture, Land Reform and Rural Development (DALRRD)and thanks to the department's geographic information system (GIS) section for developing the map for this study.

\section{References}

[1] Chen, P., \& Ye, H. (2007). Population dynamics of Bactrocera dorsalis (Diptera: Tephritidae) and analysis of factors influencing populations in Baoshanba, Yunnan, China. Entomological Science, 10(2), 141-147.

[2] Choi, K. S., Samayoa, A. C., Hwang, S. Y., Huang, Y. B., \& Ahn, J. J. (2020). Thermal effect on the fecundity and longevity of Bactrocera dorsalis adults and their improved oviposition model. PloS One, 15(7), e0235910.

[3] De Villiers, M., Hattingh, V., Kriticos, D. J., Brunel, S., Vayssières, J. F., Sinzogan, A., ... \& De Meyer, M. (2016). The potential distribution of Bactrocera dorsalis: considering phenology and irrigation patterns. Bulletin of Entomological Research, 106(1), 19-33.

[4] Drew, R. A. I., Tsuruta, K., \& White, I. M. (2005). A new species of pest fruit fly (Diptera: Tephritidae: Dacinae) from Sri Lanka and Africa. African Entomology, 13(1), 149-154.

[5] Ekesi, S., Billah, M. K., Nderitu, P. W., Lux, S. A., \& Rwomushana IV, I. (2009). Evidence for competitive displacement of Ceratitis cosyra by the invasive fruit fly Bactrocera invadens (Diptera: Tephritidae) on mango and mechanisms contributing to the displacement. Journal of Economic Entomology, 102(3), 981-991.

[6] Ekesi, S., and M. K. Billah. 2006. A Field guide to the management of economically important tephritid fruitflies in Africa. ICIPE Science Press, Nairobi, Kenya. Pp.104.

[7] Ganie, S. A., Khan, Z. H., Ahangar, R. A., Bhat, H. A., Hussain, B., \& Liu, T. (2013). Population dynamics, distribution, and species diversity of fruit flies on cucurbits in Kashmir Valley, India. Journal of Insect Science, 13(1).

[8] IPPC (2019) Pest status of Bactrocera invadens in South Africa. Pest Report Number ZAF- ZAF-48/1. Available online at https://www.ippc.int/countries/south-africa-zaf/ pest-reports/pest-status-bactrocera-invadens-south-africa (accessed March 2021).

[9] James, B. \& Schiffers, B. (2007) Exotic fly threatens African mango. Spore, 127, 6.

[10] Kriticos, D. J., Leriche, A., Palmer, D. J., Cook, D. C., Brockerhoff, E. G., Stephens, A. E. A., \& Watt, M. S. (2013). Linking climate suitability, spread rates and host-impact when estimating the potential costs of invasive pests. PLOS ONE, 8, E54861.

[11] Motswagole, R., Gotcha, N., \& Nyamukondiwa, C. (2019). Thermal biology and seasonal population abundance of Bactrocera dorsalis Hendel (Diptera: Tephritidae): implications on pest management. International Journal of Insect Science, 11, 1179543319863417.

[12] Mwatawala, M. W., White, I. M., Maerere, A. P., Senkondo, F. J., \& De Meyer, M. (2004). A new invasive Bactrocera species (Diptera: Tephritidae) in Tanzania. African entomology, 12(1), 154-156.

[13] Yang, P., Carey, J. R., \& Dowell, R. V. (1994). Temperature influences on the development and demography of Bactrocera dorsalis (Diptera: Tephritidae) in China. Environmental Entomology, 23(4), 971-974. 